\title{
Aplicação de parâmetro de resistência à fadiga para ligantes asfálticos baseado na mecânica da fratura elástico-linear
}

\section{Application of a parameter based on linear-elastic fracture mechanics to predict the fatigue resistance of asphalt binders}

\author{
Daniela Corassa Garcia', Adalberto Leandro Faxina ${ }^{2}$, Edson Denner Leonel ${ }^{3}$ \\ ${ }^{1}$ Universidade de São Paulo, São Paulo - Brasil, daniela.corassa.garcia@gmail.com \\ 2Universidade de São Paulo, São Paulo - Brasil, adalberto@usp.br \\ ${ }^{3}$ Universidade de São Paulo, São Paulo - Brasil, edleonel@sc.usp.br
}

\section{Recebido:}

11 de novembro de 2019

Aceito para publicação:

23 de janeiro de 2020

Publicado:

15 de dezembro de 2020

Editor de área:

Francisco Thiago Aragão

\section{Palavras-chaves:}

Ensaio LAS.

Mecânica da fratura.

Mecânica do contínuo.

Tolerância à fadiga.

Resistência à fadiga.

Asfaltos modificados.

Keywords:

LAS test.

Fracture mechanics.

Continuum mechanics.

Fatigue resistance.

Modified binders.

DOI:10.14295/transportes.v28i5.2090

\section{RESUMO}

Ensaios e parâmetros de fadiga para ligantes asfálticos foram desenvolvidos como alternativas ao parâmetro $G^{*} . \sin \delta$, mas também apresentam limitações. Estudos recentes indicaram que o índice de tolerância ao dano por fadiga $\left(a_{f}\right)$ do ensaio Linear Amplitude Sweep (LAS) se mostrou ineficiente para caracterizar alguns asfaltos modificados. Uma abordagem baseada na Mecânica da Fratura Elástico-Linear (MFEL) se mostrou efetiva para analisar os dados do ensaio $L A S$, inclusive nos casos em que não foi possível determinar o índice $a_{f}$. Neste trabalho, o parâmetro de tolerância à fadiga baseado na MFEL $\left(a_{M F E L}\right)$ foi avaliado quanto à aplicabilidade e representação do comportamento à fadiga. Os índices $a_{\text {MFEL }} a_{f}$ e FFL (fator de fadiga do ligante) foram comparados quanto à aplicabilidade em ensaios realizados com seis ligantes asfálticos (dois puros e quatro modificados) em três temperaturas. $O$ índice $a_{\text {MFEL }}$ pôde ser obtido em um maior número de casos que o índice $a_{f}$, indicando maior aplicabilidade do índice baseado na MFEL em relação ao índice $a_{f}$. O FFL apresentou a maior aplicabilidade dentre os três índices analisados. O $a_{\text {MFEL }}$ apresentou boa correlação com o $a_{f}$ e com o $F F L$, porém o $a_{M F E L}$ e o $a_{f}$ não apresentaram correlação significativa com o parâmetro $N_{p 20}$ do ensaio Time Sweep (TS). Dentre os índices $a_{M F E L}, a_{f}$ e $F F L$, o FFL foi o que apresentou maior correlação com o $N_{p 20}$.

\section{ABSTRACT}

Fatigue tests and parameters for asphalt binders have been developed as alternatives to the parameter $G^{*} \cdot \sin \delta$, but they also present limitations. Recent studies indicated that the fatigue damage tolerance index $\left(a_{f}\right)$ of the Linear Amplitude Sweep (LAS) test was inefficient to characterize some modified asphalts. An approach based on the Linear Elastic Fracture Mechanics (LEFM) showed to be effective to analyze the data of the LAS test, also for the cases in which was not possible to determine the $a_{f}$ index. In this work, the parameter of fatigue tolerance based on LEFM $\left(a_{\text {LEFM }}\right)$ was evaluated concerning its applicability and representation of the fatigue behavior. The indices $a_{\text {LEFM }}, a_{f}$ and BFF (binder fatigue factor) were compared in terms of their applicability to tests carried out with six asphalt binders (two neat and four modified ones) at three temperatures. It was possible to obtain the $a_{\text {LEFM }}$ index for a higher number of cases than the $\mathrm{a}_{\mathrm{f}}$ index, which would indicate a higher applicability of the index based on the LEFM when compared with the $a_{f}$ index. The BFF presented the highest applicability among the three indices. The $a_{\text {LEFM }}$ presented good correlation with $a_{f}$ and BFF, however $a_{\text {LEFM }}$ and $a_{f}$ did not present significant correlation with the parameter $N_{p 20}$ obtained from the Time Sweep (TS) test. Among the indices $a_{L E F M}, a_{f}$ and BFF, BFF presented the highest correlation with $N_{p 20 .}$ 


\section{INTRODUCÃO}

O mecanismo de fadiga em pavimentos é causado pela repetição de cargas do tráfego e pode ser considerado um dos processos de falha mais recorrentes e difíceis de mensurar (Bonnetti et al., 2002; Shenoy, 2002). Nesse contexto, a utilização de ensaios e parâmetros de fadiga para ligantes asfálticos apresenta-se como uma alternativa para comparação e seleção de materiais, uma vez que a resistência à fadiga do ligante influencia de forma significativa o desempenho à fadiga da mistura asfáltica (Anderson e Kennedy, 1993; Bahia et al., 2001a; Hintz et al., 2011; Johnson, 2010; Soenen et al., 2003).

Estudos indicaram que o critério baseado no parâmetro $G^{*}$.sen $\delta$ (módulo de cisalhamento dinâmico dividido pelo seno do ângulo de fase) da especificação Superpave (Superior Performing Pavements) não seria apropriado para caracterização do comportamento à fadiga de ligantes asfálticos sob cargas cíclicas. Os ensaios para determinação do módulo complexo e ângulo de fase são executados em uma faixa de deformação baixa, na qual o material está no regime de viscoelasticidade linear, ao passo que o processo de fadiga se dá na região de viscoelasticidade não-linear, onde ocorrem altas deformações (Bahia et al., 2001a; Sabouri et al., 2018; Shenoy, 2002).

Durante o NCHRP 9-10 (National Cooperative Highway Research Program), Bahia et al. (2001a) propuseram um ensaio de varredura de tempo (time sweep - TS), caracterizado pela aplicação cíclica de uma configuração específica de tensão (ou deformação), temperatura e frequência, e adotaram o parâmetro de falha $N_{p}$. Apesar de apresentar boa correlação com o desempenho à fadiga de misturas asfálticas (Anderson et al., 2001; Bahia et al., 2001b), o ensaio não apresentou boa repetibilidade, além da possibilidade de ensaios com duração excessivamente longa (Anderson et al., 2001; Bahia et al., 2010).

Na sequência, Johnson (2010) desenvolveu o ensaio de varredura de amplitude linear (linear amplitude sweep - $L A S$ ), que é um procedimento acelerado em que ao material é levado à fadiga pela aplicação de incrementos de carga. 0 método de análise baseou-se na teoria da mecânica do dano contínuo em meio viscoelástico (viscoelastic continuum damage - VECD), que considera a hipótese de continuidade da amostra, para obtenção da curva $C$ versus $S$ e dos parâmetros que permitem traçar a curva de fadiga dos materiais.

Logo em seguida, Hintz (2012) observou amostras ensaiadas após o ensaio LAS e verificou a ocorrência de macrofissurações e consequente diminuição do raio da amostra, as quais seriam responsáveis pela diminuição da rigidez do material. Essa hipótese embasou a adoção de um modelo de análise baseado na fratura, com a consideração do comprimento da fissura e da taxa de crescimento da fissura para adoção do índice $a_{f}$ (comprimento de fissura na falha). Hintz (2012) também propôs uma alteração importante no formato da aplicação da carga, que passou a ser aplicada com incremento linear, no lugar de incrementos discretos. Por conta desta modificação, o ensaio passou a ser conhecido como ensaio LAS modificado.

Ao aplicar o procedimento proposto por Hintz (2012) a uma variedade de asfaltos modificados envelhecidos a curto e a longo prazos, Nuñez (2013) não obteve sucesso ao aplicar o método de análise baseado na fratura a alguns daqueles asfaltos modificados. Na tentativa de estabelecer um índice que pudesse ser obtido nos casos em que não foi possível mensurar o índice $a_{f}$, Nuñez et al. (2016) sugeriram a adoção de um índice de falha baseado na mecânica da fratura elástico-linear (MFEL), com o objetivo de investigar o uso da teoria da mecânica da fratura 
para representar o fenômeno de fadiga em ligantes e estabelecer um critério de falha com maior aplicabilidade que o índice $a_{f}$.

0 trabalho desenvolvido por Nuñez et al. (2016) utiliza uma modelagem numérica que utiliza o programa FRANC2D/L (FRacture ANalysis Code-2D with Layers) para simulação do ensaio LAS, por meio de uma propagação de fissuras na qual os incrementos de fissura possuem o mesmo valor. 0 programa adotado neste estudo foi desenvolvido durante o NASA Airframe Structural Integrity Program (NASIP) pela Universidade de Cornell e utiliza o método dos elementos finitos para modelar a propagação de fissuras, bem como a integral J e o critério da máxima tensão circunferencial para o cálculo do fator de intensidade de tensão considerando o regime elástico.

Segundo Nuñez et al. (2016), os resultados da abordagem sugerida apresentaram tendência similar a resultados de ensaio $T S$, apesar do trabalho não apresentar os resultados de correlação. Concluiu-se que o índice proposto poderia ser um índice prático para comparação de materiais, pincipalmente nos casos em que o índice $a_{f}$ não pudesse ser obtido. Porém, o trabalho não comparou a abordagem baseada na MFEL com outras formas de análise do ensaio LAS. Mais recentemente, Safaei e Castorena (2016) indicaram que ensaios com materiais que apresentassem valores de $G^{*}$ inferiores a $12 \mathrm{MPa}$ e superiores a $60 \mathrm{MPa}$ poderiam apresentar problemas de instabilidade e de perda de aderência do material às placas paralelas do reômetro, o que também não foi analisado no trabalho desenvolvido por Nuñez et al. (2016).

0 objetivo do presente trabalho é utilizar incrementos de fissura calculados com base nos resultados do ensaio $L A S$ para simulação do ensaio com o programa FRANC2D/L, no intuito de melhor representar o processo de trincamento que ocorre no material, e comparar a abordagem baseada na MFEL com a abordagem baseada na fratura e com a abordagem baseada na teoria $V E C D$. Além disso, a faixa de valores de $G^{*}$ será avaliada de forma a verificar se problemas de instabilidade ou perda de aderência são os responsáveis pela menor aplicabilidade do índice $a_{f}$.

\section{ENSAIOS E PARÂMETROS DE FADIGA PARA LIGANTES ASFÁLTICOS}

\subsection{Varredura de tempo - Time Sweep (TS)}

Bahia et al. (2001a) aplicaram o ensaio de varredura de tempo a ligantes asfálticos e o critério de ruptura foi arbitrado como o número de ciclos completados no ponto onde há uma redução de 50\% do módulo complexo inicial $G^{*}$. Pesquisas posteriores indicaram que esse parâmetro não tem relação com o acúmulo de dano na amostra (Anderson et al., 2001) e outras definições de ruptura foram propostas, como a baseada no conceito de energia dissipada. 0 parâmetro baseado neste conceito inclui a taxa de energia dissipada (DER - Dissipated Energy Ratio) e a taxa de acúmulo de dano (Anderson et al., 2001). A Equação 1 é utilizada para o cálculo da energia dissipada por ciclo $\left(W_{i}\right)$ para um material viscoelástico, onde $\sigma_{i}$ é a tensão no ciclo $i, \varepsilon_{i}$ é a deformação no ciclo $i$ e $\delta_{i}$ é o ângulo de fase no ciclo $i$. A energia dissipada acumulada até o ciclo n $\left(W_{T n}\right)$ é definida de acordo com a Equação 2. A taxa de energia dissipada (DER), definida por Pronk e Hopman (1991) e usada como critério de fadiga, é definida pela Equação 3.

$$
\begin{gathered}
\mathrm{W}_{\mathrm{i}}=\pi \sigma_{\mathrm{i}} \varepsilon_{\mathrm{i}} \operatorname{sen} \delta_{\mathrm{i}} \\
\mathrm{W}_{\mathrm{T}_{\mathrm{n}}}=\sum_{\mathrm{i}=1}^{\mathrm{n}} \mathrm{W}_{\mathrm{i}} \\
\mathrm{DER}=\frac{\mathrm{W}_{\mathrm{T}_{\mathrm{n}}}}{\mathrm{W}_{\mathrm{n}}}
\end{gathered}
$$

Bahia et al. (2001a) utilizaram como critério de ruptura o parâmetro $N_{p}$, que corresponde ao número de ciclos até o qual a amostra não apresenta dano, isto é, até o ciclo em que o coeficiente angular da curva DER versus número de ciclos é constante. Bonnetti et al. (2002) avaliaram os 
critérios de falha $N_{p 10}$ e $N_{p 20}$ (número de ciclos associado a um desvio de $10 \%$ e $20 \%$, respectivamente, em relação à linha de igualdade $D E R=N$ ) e concluiu-se que o parâmetro $N_{p 20}$ se mostrou mais claramente definido, menos afetado pelo erro experimental e menos sensível ao tipo de carregamento, além de caracterizar melhor o comportamento à fadiga dos ligantes asfálticos. 0 critério $N_{p}$ também foi interpretado como o número de ciclos onde tem início a propagação de trincas. Esta grandeza pode ser determinada usando a Equação 4 (Bahia et al., 2001a; Bonnetti et al., 2002).

$$
20 \%=\frac{\mathrm{DER}_{\mathrm{N}_{\mathrm{p} 20}-\mathrm{N}_{\mathrm{p} 20}}}{\mathrm{~N}_{\mathrm{p} 20}} \cdot 100
$$

\subsection{Varredura de amplitude linear - Linear Amplitude Sweep (LAS)}

\subsubsection{Aplicação da teoria da mecânica do dano contínuo em meio viscoelástico (VECD)}

Johnson (2010) aplicou a teoria do dano contínuo em meio viscoelástico para ajustar um modelo de fadiga do ligante asfáltico. Esse procedimento está inserido no projeto de norma AASHTO TP 101-14 (AASHTO, 2014). Misturas e ligantes asfálticos demonstraram uma boa relação entre a carga aplicada e a vida de fadiga $\left(N_{f}\right)$ (Hintz et al., 2011; Johnson, 2010; Martins, 2014; Nascimento et al., 2014) dada pela Equação 5, onde os parâmetros $A$ e $B$ são derivados do ajuste e $\gamma_{\text {máx }}$ é a deformação máxima esperada para uma dada estrutura do pavimento. 0 parâmetro $B$ é calculado de acordo com a Equação 6, sendo $\alpha$ dado pela Equação 7, em que $m$ é a inclinação da reta do gráfico log-log do módulo de armazenamento $\left(G^{*} \cdot \cos \delta\right.$ ) versus frequência.

$$
\begin{gathered}
\mathrm{N}_{\mathrm{f}}=\mathrm{A}\left(\gamma_{\text {máx }}\right)^{\mathrm{B}} \\
\mathrm{B}=2 \alpha \\
\alpha=\frac{1}{m}
\end{gathered}
$$

0 parâmetro $A$ é calculado de acordo com a Equação 8, onde $f$ é a frequência $(10 \mathrm{~Hz}), D_{f}$ é o valor de $D(t)$ na falha, definido com a Equação 14, e $k$ é definido com a Equação 9:

$$
\begin{gathered}
A=\frac{f\left(D_{f}\right)^{k}}{k\left(\pi C_{1} C_{2}\right)^{\alpha}} \\
k=1+\left(1-C_{2}\right) \alpha
\end{gathered}
$$

0 acúmulo de dano na amostra $(D(t))$ é calculado segundo a Equação 10, adaptada de Kim et al., (2006):

Onde:

$$
\mathrm{D}(\mathrm{t}) \cong \sum_{1=\mathrm{i}}^{\mathrm{N}}\left[\pi \gamma_{0}^{2}\left(\mathrm{C}_{\mathrm{i}-1}-\mathrm{C}_{\mathrm{i}}\right)\right]^{\frac{\alpha}{1+\alpha}}\left(\mathrm{t}_{\mathrm{i}}-\mathrm{t}_{\mathrm{i}-1}\right)^{\frac{\alpha}{1+\alpha}}
$$

$$
C(t)=\frac{/ G^{*} /(t)}{\mid G^{*} / \text { inicial }}
$$

Onde $\left|G^{*}\right|$ é o módulo de cisalhamento complexo em MPa, $\alpha$ dado pela Equação 7 e $t$ é o tempo de ensaio em segundos.

A relação entre $C(t)$ e $D(t)$ pode ser ajustada pelo modelo presente na Equação 12, onde o valor de $C_{0}$ é 1 e os valores iniciais de $C_{1}$ e $C_{2}$ são obtidos da linearização do modelo dado na Equação 13, adaptado de Hintz et al., 2011. O valor de $D(t)$ na falha, $D_{f}$, é definido como o $D(t)$ que corresponde a redução no $G^{*}$ inicial no pico de tensão e é calculado de acordo com a Equação 14.

$$
\begin{gathered}
C(t)=C_{0}-C_{1}(D)^{C_{2}} \\
\log \left(C_{0}-C(t)\right)=\log \left(C_{1}\right)+C_{2} \cdot \log (D(t))
\end{gathered}
$$




$$
\mathrm{D}_{\mathrm{f}}=\left(\frac{\mathrm{C}_{0}-\mathrm{C}_{\text {pico de tensão }}}{\mathrm{C}_{1}}\right)^{1 / \mathrm{C}_{2}}
$$

0 parâmetro $A$ retrata a variação da integridade do material $\left(G^{*}\right)$ em função do dano acumulado. Johnson (2010) admitiu genericamente que a falha ocorre quando o material atingir uma integridade de 35\% da sua integridade original. Já Martins (2014) usou um critério de ruptura diferente do proposto por Johnson (2010), tendo adotado um parâmetro denominado $C_{f}$, correspondente ao valor de integridade do material no ponto onde começa a rápida propagação da fissura. 0 ajuste da curva da Equação 10 é realizado com os dados até o valor de $C_{f}$, dado que após este ponto o crescimento da fissura é instável. Por sua vez, o parâmetro $B$ depende exclusivamente de $\alpha$, que representa a inclinação do gráfico $G^{*} \cdot \cos \delta$ versus frequência, obtido do ensaio de varredura de frequência. Com a diminuição dos valores de $B$, a declividade da curva de fadiga diminui, indicando que o ligante asfáltico se torna menos sensível ao aumento da amplitude de deformação e, consequentemente, sua vida de fadiga aumenta.

Martins (2014) também sugeriu a adoção de um índice derivado da curva de fadiga obtida por meio da abordagem baseada na teoria $V E C D$, o qual é denominado fator de fadiga do ligante ( $F F L$ ). O FFL representa a área sob a curva log-log de fadiga para deformações de 1,25\% e 2,50\%, conforme ilustrado na Figura 1 e é calculado de acordo com a Equação 15. A definição destes limites de deformação na escala do ligante asfáltico se baseou nas considerações feitas por Underwoord (2011) que assumiu que deformações da ordem de 200 microstrains a 400 microstrains são típicas de pavimentos e que o ligante asfáltico está submetido a uma deformação da ordem de 122,5 vezes maior que a sofrida pela mistura asfáltica como um todo.

$$
\mathrm{FFL}=\frac{\left(\log \left(\mathrm{N}_{\mathrm{f}, 1,25 \%}\right)+\log \left(\mathrm{N}_{\mathrm{f}, 2,5 \%}\right)\right)}{2} \cdot(\log (0,025)-\log (0,0125))
$$

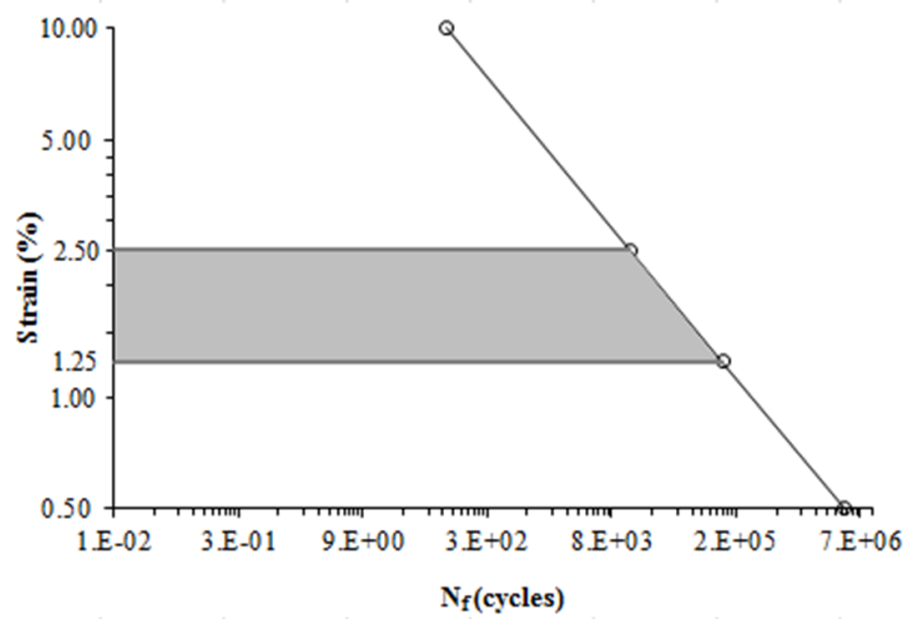

Figura 1. Área correspondente ao critério FFL na curva de fadiga (adaptado de Martins, 2014)

\subsubsection{Aplicação do modelo baseado na fratura}

O procedimento proposto por Hintz (2012) permite estimar o comprimento da fissura (a) e a taxa de crescimento da fissura $(d a / d N)$ - Equações 16 e 17, em que $a$ é o comprimento da fissura, $T$ é o torque, $\phi$ é o ângulo de deflexão e $T_{i}$ e $\phi_{i}$ são o torque e o ângulo de deflexão no ciclo $i$, respectivamente. 


$$
\begin{gathered}
\mathrm{a}=4-4 \cdot\left(\frac{\mathrm{T} / \phi}{\mathrm{T}_{\mathrm{i}} / \phi_{\mathrm{i}}}\right) \\
\left(\frac{\mathrm{da}}{\mathrm{dN}}\right)=\left(\frac{\Delta \mathrm{a}}{\Delta \mathrm{N}}\right)
\end{gathered}
$$

0 critério de falha utilizado por Hintz (2012) foi o comprimento da fissura na falha ( $a_{f}$ ). A curva de taxa de crescimento da fissura por comprimento de fissura apresenta dois picos. Após o primeiro pico, há uma rápida taxa de crescimento da fissura que representaria o ponto a partir do qual a amostra apresentará rápida propagação de fissuras (Figura 2). Esse parâmetro é utilizado como índice para comparação de materiais. Segundo Hintz (2012), um material que suporta maior fissuração antes que se inicie a propagação rápida das trincas deve apresentar um maior tamanho da fissura na falha. Esta característica indicaria sua maior capacidade em resistir ao dano por fadiga.

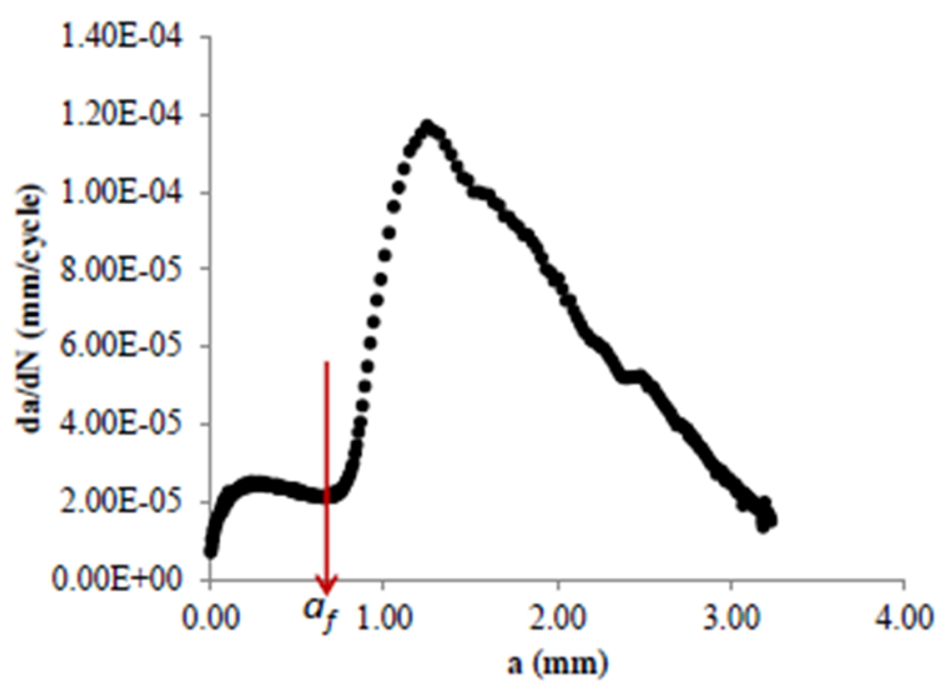

Figura 2. Parâmetro de falha $a_{f}$ para o ensaio LAS modificado (Hintz, 2012)

\subsubsection{Abordagem baseada na mecânica da fratura elástico-linear (MFEL)}

A abordagem sugerida por Nuñez et al. (2016) utiliza os conceitos da mecânica da fratura elástico-linear (MFEL) e adota como critério de ruptura o ponto de inflexão da curva de fator de intensidade de tensão $(K)$ versus comprimento da fissura $(a)$, como mostra a Figura 3. 0 fator de intensidade de tensão indica a magnitude de tensão presente na ponta da fissura, de modo que a redução da magnitude da tensão indicaria o crescimento rápido da fissura. 0 estudo comparou uma solução analítica e uma solução numérica para análise do ensaio LAS. A solução analítica foi feita com base na Equação 18, utilizada para obtenção do fator de intensidade de tensão para o modo de fissura de abertura (modo I) para o problema de Griffith (Griffith, 1921; Irwin, 1957), em que $K_{I}$ é o fator de intensidade de tensão para o modo I, $\sigma$ é a tensão aplicada e $a$ é o comprimento da fissura. Foram utilizados os comprimentos de fissura obtidos por meio da Equação 16 aplicada aos resultados do ensaio.

$$
\mathrm{K}_{\mathrm{I}}=\sigma \sqrt{\pi \mathrm{a}}
$$






Figura 3. Parâmetro de falha sugerido para o ensaio LAS

A solução numérica foi obtida por meio da modelagem do ensaio $L A S$ no software FRANC2D/L. A fissuração foi reproduzida por meio de um incremento de fissura constante. 0 fator de intensidade de tensão equivalente $\left(K_{e q}\right)$ foi calculado por meio da Equação 19, que considera o fator de intensidade para o modo de fissura de abertura (modo I) e o fator de intensidade para o modo de fissura de deslocamento (modo II). $K_{I}$ e $K_{I I}$ são os fatores de tensão para os modos de fratura I e II, respectivamente, e $\theta_{p}$ é o ângulo de dobra da fissura. 0 ponto de falha foi dado pelo ponto máximo da curva $K e q$ versus $a$.

$$
\mathrm{K}_{\mathrm{eq}}=\mathrm{K}_{\mathrm{I}} \cos ^{3}\left(\frac{\theta_{\mathrm{P}}}{2}\right)-3 \mathrm{~K}_{\mathrm{II}} \cos ^{2}\left(\frac{\theta_{\mathrm{P}}}{2}\right) \operatorname{sen}\left(\frac{\theta_{\mathrm{P}}}{2}\right)
$$

\section{MATERIAIS E MÉTODOS}

\subsection{Materiais}

Foram utilizados ligantes asfálticos puros e modificados para aplicação dos diferentes métodos de análise do ensaio LAS. Os ligantes foram fornecidos por dois distribuidores de asfaltos brasileiros, sendo dois ligantes puros e quatro modificados. Os materiais serão referenciados pelas letras de $\mathrm{A}$ a $\mathrm{F}$, conforme indicado a seguir, de modo a omitir seus nomes comerciais.
(A) CAP 30/45 - PG 70-16;
(B) CAP 50/70 - PG 64-22;
(C) Asfalto modificado com polímero elastomérico comercial 1 - PG 76-16;
(D) Asfalto modificado com polímero elastomérico comercial 2 - PG 76-22;
(E) Asfalto de alto módulo comercial - PG 82-16;
(F) Asfalto modificado com polímero elastomérico comercial 3 - PG 70-22.

\subsection{Ensaios de laboratório}

As amostras de cada ligante foram submetidas a envelhecimento de curto prazo na estufa RTFO (Rolling Thin Film Oven), segundo o procedimento ASTM D 2872 - 12e1, e posteriormente foram submetidas a envelhecimento de longo prazo, utilizando a estufa de vaso pressurizado, segundo o procedimento ASTM D 6521-18. Tanto a varredura de tempo quanto a varredura de amplitude linear foram realizadas no reômetro de cisalhamento dinâmico (dynamic shear rheometer - DSR) da marca TA Instruments, modelo AR2000ex, empregando a geometria de placas paralelas de $8 \mathrm{~mm}$ de diâmetro com distância de $2 \mathrm{~mm}$ entre placas. 
As varreduras de tempo foram realizadas em condições fixas: frequência de $10 \mathrm{rad} / \mathrm{s}$, percentual de deformação de $10 \%$ e temperatura de $25^{\circ} \mathrm{C}$. A temperatura de $25{ }^{\circ} \mathrm{C}$ vem sendo utilizada por diversos pesquisadores em ensaios de fadiga (Anderson et al., 2001; Bahia e Anderson, 1995; Bonnetti et al., 2002; Pamplona et al., 2014). Outras pesquisas empregaram temperaturas ligeiramente inferiores a $25^{\circ} \mathrm{C}$ : Martins (2014) realizou os ensaios $L A S$ a $19{ }^{\circ} \mathrm{C}$, a norma AASHTO TP 101-14 recomenda a adoção da temperatura intermediária do grau de desempenho PG do ligante asfáltico e Safaei e Castorena (2016) recomendam o uso de uma temperatura $4{ }^{\circ} \mathrm{C}$ abaixo da temperatura intermediária do PG do ligante asfáltico. 0 ensaio de varredura de amplitude linear é dividido em duas etapas: (1) uma varredura de frequência entre 0,1 e $30 \mathrm{~Hz}$ é realizada para determinar as propriedades reológicas na região de viscoelasticidade linear, utilizando a amplitude de deformação de 0,1\% e (2) uma varredura de amplitude de deformação entre $0,1 \%$ e $30 \%$ é realizada a uma frequência fixa de $10 \mathrm{~Hz}$, com incremento linear da deformação, conforme adaptação feita por Hintz et al. (2011).

\subsection{Análise dos resultados}

A Figura 4 apresenta um fluxograma com os ensaios e abordagens de análise utilizados neste trabalho. Os resultados do ensaio de varredura de tempo foram analisados com base no conceito de taxa de energia dissipada, com adoção do parâmetro $N_{p 20}$, como descrito no item 2.1. A abordagem baseada na teoria do dano contínuo em meio viscoelástico foi utilizada na análise dos resultados dos ensaios de varredura de amplitude linear para obtenção do índice $F F L$, conforme o item 2.2.1. Para isso, foi considerada a integridade real do material na ruptura, com base no ponto de máximo da curva de tensão versus deformação. 0 índice $a_{f}$ foi obtido por meio da abordagem baseada na fratura, conforme o item 2.2.2, e a curva de torque versus comprimento da fissura foi utilizada para verificação da falha do material, como indicado por Martins (2014). 0 índice $a_{M F E L}$ foi obtido por meio da aplicação da abordagem baseada na mecânica da fratura elástico-linear, conforme o item 3.3.

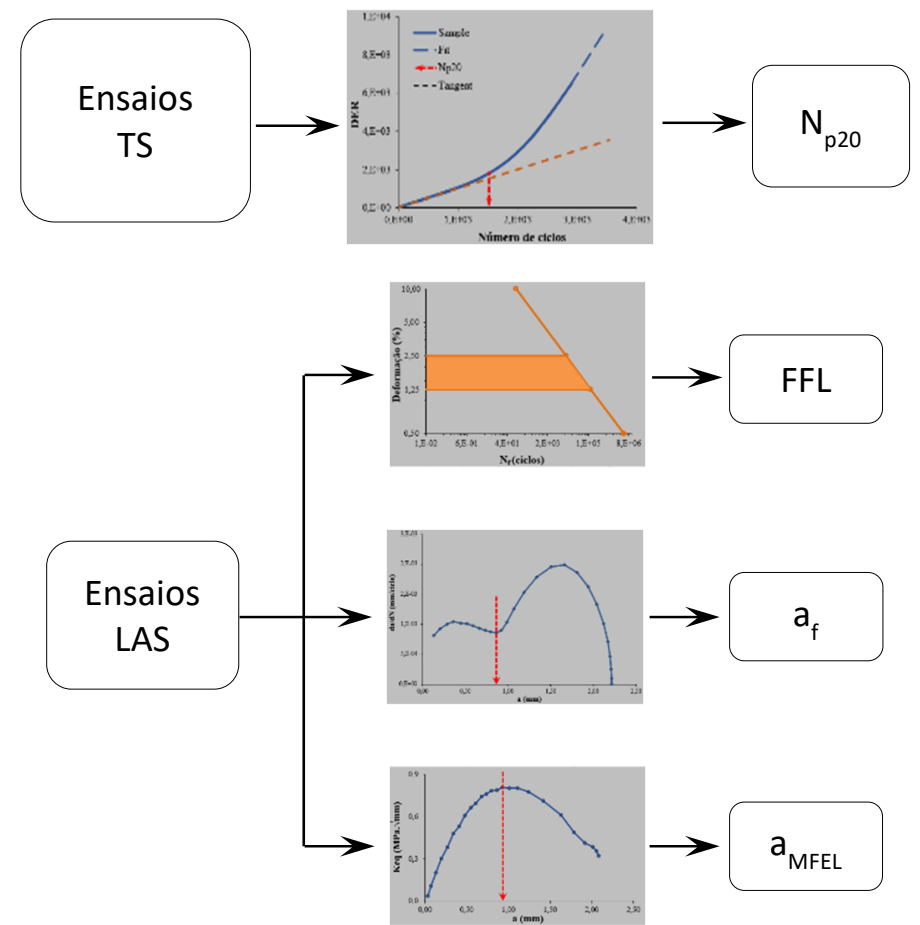

Figura 4. Esquema com os ensaios e abordagens de análise utilizadas 


\subsection{Abordagem baseada na mecânica da fratura elástico-linear (MFEL)}

0 procedimento adotado na abordagem baseada na mecânica da fratura elástico-linear se baseou na modelagem numérica sugerida por Nuñez et al. (2016), porém utilizou os incrementos de fissura calculados com base nos resultados do ensaio LAS. O software FRANC2D/L (FRANC2D/L, 2016) foi utilizado para simulação do ensaio e obtenção do fator de intensidade de tensão equivalente $\left(K_{e q}\right)$. A simulação do ensaio é feita em dois passos: geração da geometria e da malha com o uso do software CASCA e simulação do ensaio no software FRANC2D/L. A geometria construída no software CASCA possui quatro unidades no comprimento e duas unidades na altura para representar metade da geometria da amostra, como mostra a Figura 5. A malha, composta de dez elementos finitos quadrangulares no eixo vertical e vinte elementos finitos quadrangulares no eixo horizontal, foi escolhida após estudo de convergência de malha além de ser a mesma utilizada por Nuñez et al. (2016). A lateral esquerda e o lado inferior da seção foram fixados nos eixos x e y de forma a simular a adesão da amostra à placa. Uma tensão de cisalhamento máxima unitária foi imposta à seção para simular a torção aplicada à amostra durante o ensaio de varredura de tempo, como mostra a Figura 6.
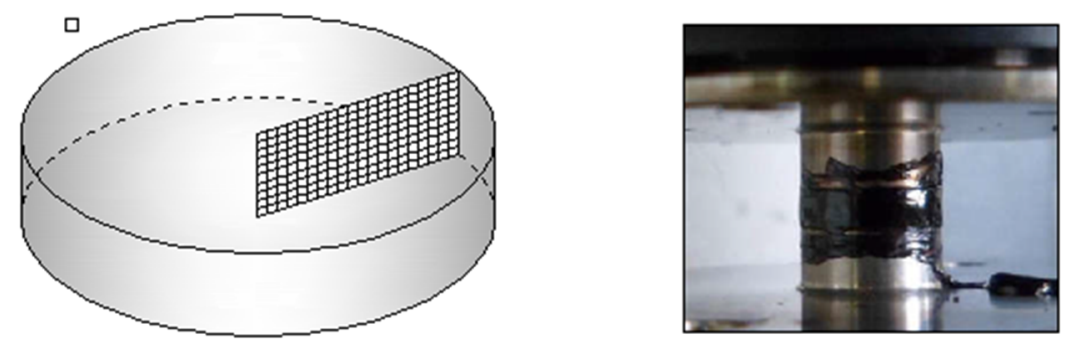

Figura 5. Amostra e seção longitudinal utilizada para simulação (adaptado de Nuñez et al., 2016)

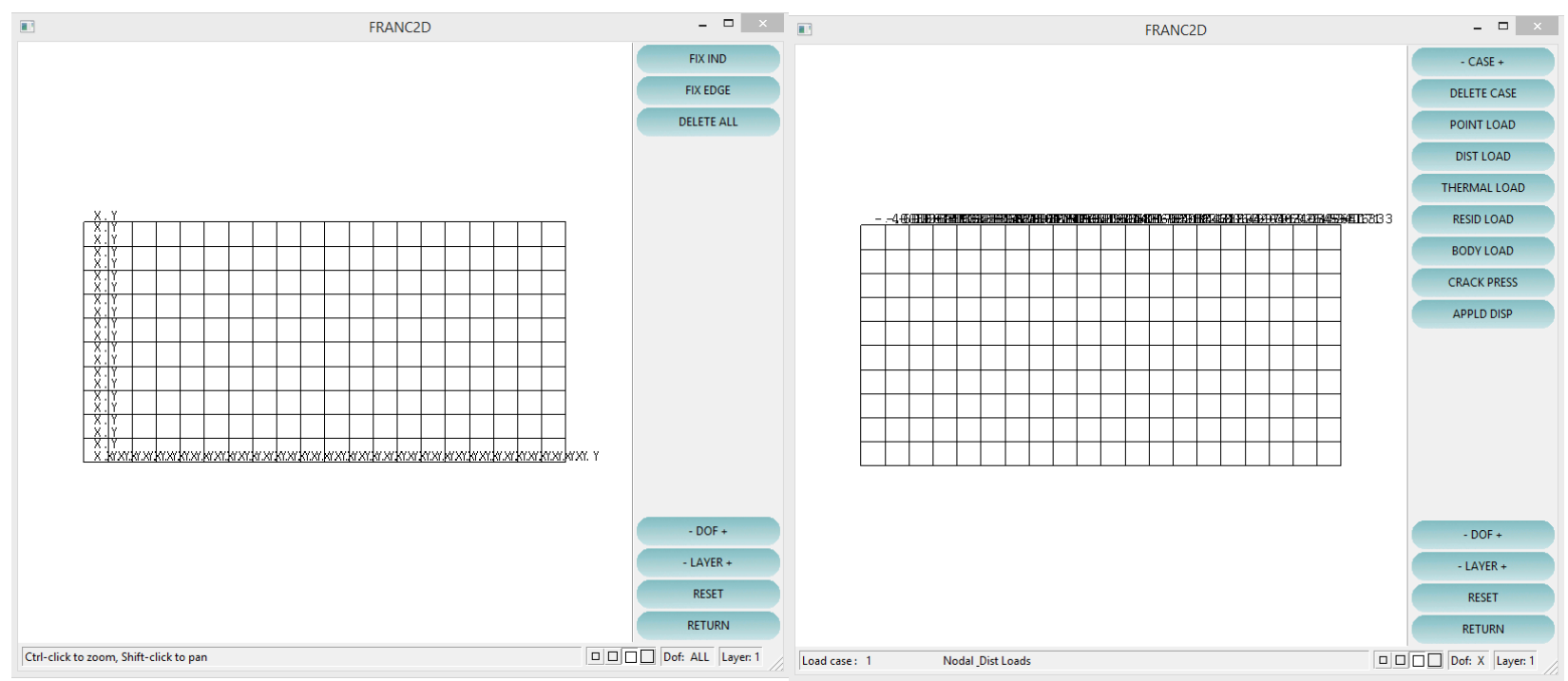

Figura 6. Seção sob condições de fixação e carregamento no programa FRANC2D/L

O software FRANC2D/L utiliza o coeficiente de Poisson e o módulo de elasticidade para caracterizar o material. A análise elástico-linear representa uma simplificação do comportamento viscoelástico do material e foi utilizada nesta pesquisa a fim de viabilizar a avaliação de uma aplicação simplificada da mecânica da fratura. Estudos futuros podem tomar como base os resultados aqui apresentados e avaliar ferramentas mais apropriadas. 0 coeficiente de Poisson 
adotado foi 0,49 (Anderson et al., 1994; Christensen Jr. e Anderson, 1992), já que o limite de elasticidade é 0,50 , e o módulo de elasticidade foi estimado com base no valor de $G^{*}$ a $10 \mathrm{rad} / \mathrm{s}$ para cada material e condição ensaio por meio da Equação 20, em que $E$ é o módulo de elasticidade, $G$ é o módulo de cisalhamento e $v$ é o coeficiente de Poisson (Timoshenko e Gere, 1983).

$$
\mathrm{E}=2 \mathrm{G} .(1+v)
$$

A simulação no software FRANC2D/L seguiu a seguinte rotina: (i) carregamento no FRANC2D/L do arquivo gerado no CASCA; (ii) definição das propriedades do material e das condições de contorno; (iii) análise da configuração criada por meio da opção ANALYSIS no menu principal; (iv) Simulação do crescimento da fissura com a opção MODIFY; (v) obtenção dos fatores de intensidade de tensão para os modos I e II na opção POST PROCESS; (vi) obtenção do fator de intensidade de tensão equivalente por meio da Equação 19 para cada passo de carregamento. Os resultados de $K_{e q}$ e comprimento de fissura são plotados e o ponto máximo é considerado como critério de falha do material $\left(a_{M F E L}\right)$. A rotina descrita encontra-se esquematizada na Figura 7.



Figura 7. Rotina para obtenção do índice $a_{M F E L}$

\section{APRESENTAÇÃO E ANÁLISE DOS RESULTADOS}

Foram realizados pelo menos dois ensaios LAS para cada material e temperatura e a variabilidade entre resultados foi controlada, de modo a minimizar a heterogeneidade dos resultados. A Tabela 1 apresenta os valores médios de módulo de cisalhamento complexo, dos índices $a_{M F E L}, a_{f}$ e FFL encontrados para os ensaios $L A S$ realizados com os seis materiais nas temperaturas de $15{ }^{\circ} \mathrm{C}, 25^{\circ} \mathrm{C}$ e $35^{\circ} \mathrm{C}$, além das respectivas variabilidades encontradas. Os valores de $G^{*}$ fora da faixa de $12 \mathrm{MPa}$ a $60 \mathrm{MPa}$ (Safaei e Castorena, 2016) estão destacados na Tabela 1. Em alguns casos, não foi possível obter o valor médio dos índices, uma vez que os resultados individuais não permitiram a aplicação dos critérios de ruptura. A Figura 8 apresenta os casos em que não foi possível a obtenção do índice $a_{f}$ e foi possível a obtenção do índice $a_{M F E L \text {. Nesses }}$ casos, o ponto de inflexão da curva de $d a / d N$ vs. a não se apresentou de forma definida, ao passo que foi possível obter o ponto máximo da curva de $K_{e q}$ vs. $a$. Estão apresentados os gráficos de $d a / d N$ vs. $a$ e $K_{e q}$ vs. $a$ para os ensaios realizados com os materiais A (CAP 30/45) a $35{ }^{\circ}$ C, B (CAP 50/70) a $35^{\circ} \mathrm{C}, \mathrm{C}$ (asfalto modificado com polímero elastomérico) a $25^{\circ} \mathrm{C}$ e $\mathrm{E}$ (asfalto de alto módulo) a $15{ }^{\circ} \mathrm{C}$. 
Tabela 1 - Resultados de $G^{*}$, dos índices $a_{M F E L}, a_{f}$ e $F F L$ e dos parâmetros $A$ e $B$ do modelo de fadiga

\begin{tabular}{|c|c|c|c|c|c|c|c|c|}
\hline Material & $\mathrm{T}(\stackrel{\mathrm{o}}{ } \mathrm{C})$ & Medidas & $G^{*}(\mathrm{MPa})$ & $\begin{array}{l}a_{M F E L} \\
(\mathrm{~mm})\end{array}$ & $a_{f}(\mathrm{~mm})$ & $A$ & $B$ & FFL \\
\hline \multirow{6}{*}{ A } & \multirow{2}{*}{$15^{\circ} \mathrm{C}$} & média & 84,90 & - & - & - & - & - \\
\hline & & variabilidade (\%) & 4,8 & - & - & - & - & - \\
\hline & \multirow{2}{*}{$25^{\circ} \mathrm{C}$} & média & 25,61 & 0,89 & 0,81 & 89.273 & $-3,32$ & 1,24 \\
\hline & & variabilidade (\%) & 3,0 & 3,2 & 3,9 & 42,9 & 2,0 & 4,3 \\
\hline & \multirow{2}{*}{$35^{\circ} \mathrm{C}$} & média & 5,80 & 0,79 & - & 200.134 & $-2,61$ & 1,39 \\
\hline & & variabilidade (\%) & 3,7 & 1,8 & - & 46,4 & 2,0 & 4,8 \\
\hline \multirow{6}{*}{ B } & \multirow{2}{*}{$15^{\circ} \mathrm{C}$} & média & 57,57 & - & - & 160.353 & $-4,1$ & 1,26 \\
\hline & & variabilidade (\%) & 5,9 & - & - & 31,8 & 1,1 & 3,5 \\
\hline & \multirow{2}{*}{$25^{\circ} \mathrm{C}$} & média & 15,27 & 1,01 & 0,83 & 193.360 & $-3,08$ & 1,36 \\
\hline & & variabilidade (\%) & 0,5 & 2,8 & 6,2 & 13,7 & 1,1 & 1,5 \\
\hline & \multirow{2}{*}{$35^{\circ} \mathrm{C}$} & média & 3,22 & 0,77 & - & 279.412 & $-2,58$ & 1,45 \\
\hline & & variabilidade (\%) & 4,3 & 2,8 & - & 25,3 & 0,6 & 2,2 \\
\hline \multirow{6}{*}{ C } & \multirow{2}{*}{$15^{\circ} \mathrm{C}$} & média & 29,23 & - & - & 574.199 & $-4,39$ & 1,33 \\
\hline & & variabilidade (\%) & 4,7 & - & - & 129,8 & 1,5 & 13,5 \\
\hline & \multirow{2}{*}{$25^{\circ} \mathrm{C}$} & média & 10,07 & 1,26 & - & 12.204 .662 & $-3,51$ & 1,44 \\
\hline & & variabilidade (\%) & 3,2 & 1,1 & - & 114,1 & 9,8 & 1,6 \\
\hline & \multirow{2}{*}{$35^{\circ} \mathrm{C}$} & média & 2,01 & - & - & - & - & - \\
\hline & & variabilidade (\%) & 1,4 & - & - & - & - & - \\
\hline \multirow{6}{*}{ D } & \multirow{2}{*}{$15^{\circ} \mathrm{C}$} & média & 41,67 & 1,19 & 1,00 & 215.325 & $-3,95$ & 1,30 \\
\hline & & variabilidade (\%) & 7,8 & 1,8 & 37,7 & 40,5 & 3,2 & 3,7 \\
\hline & \multirow{2}{*}{$25^{\circ} \mathrm{C}$} & média & 15,37 & 1,28 & 1,13 & 699.809 & $-3,33$ & 1,48 \\
\hline & & variabilidade (\%) & 3,4 & 1,1 & 0,4 & 16,3 & 6,1 & 0,2 \\
\hline & \multirow{2}{*}{$35^{\circ} \mathrm{C}$} & média & 2,42 & - & 0,56 & 3.492 .976 & $-2,95$ & 1,75 \\
\hline & & variabilidade (\%) & 6,5 & - & 6,8 & 11,2 & 1,0 & 0,7 \\
\hline \multirow{6}{*}{$E$} & \multirow{2}{*}{$15^{\circ} \mathrm{C}$} & média & 73,34 & 1,20 & - & 647.914 & $-5,15$ & 1,36 \\
\hline & & variabilidade (\%) & 5,8 & 0,6 & - & 17,5 & 0,8 & 1,8 \\
\hline & \multirow{2}{*}{$25^{\circ} \mathrm{C}$} & média & 25,14 & 1,10 & 0,94 & 1.480 .909 & $-4,43$ & 1,52 \\
\hline & & variabilidade (\%) & 3,6 & 6,4 & 14,0 & 54,6 & 0,5 & 5,1 \\
\hline & \multirow{2}{*}{$35^{\circ} \mathrm{C}$} & média & 7,39 & 1,13 & 0,89 & 7.282 .819 & $-3,86$ & 1,77 \\
\hline & & variabilidade (\%) & 9,2 & 10,0 & 22,4 & 60,3 & 4,0 & 5,4 \\
\hline \multirow{6}{*}{$\mathrm{F}$} & \multirow{2}{*}{$15^{\circ} \mathrm{C}$} & média & 54,62 & 1,20 & 1,22 & 113.225 & $-3,87$ & 1,23 \\
\hline & & variabilidade (\%) & 2,3 & 4,5 & 11,6 & 15,3 & 3,0 & 2,2 \\
\hline & \multirow{2}{*}{$25^{\circ} \mathrm{C}$} & média & 14,59 & 1,11 & 0,99 & 360.357 & $-3,13$ & 1,44 \\
\hline & & variabilidade (\%) & 5,7 & 2,9 & 2,1 & 23,6 & 1,3 & 2,3 \\
\hline & \multirow{2}{*}{$35^{\circ} \mathrm{C}$} & média & 2,88 & 1,01 & 0,52 & 1.239 .486 & $-2,76$ & 1,63 \\
\hline & & variabilidade (\%) & 10,1 & 34,1 & 15,7 & 19,0 & 1,3 & 1,5 \\
\hline
\end{tabular}

O número de casos em que foi possível obter o valor médio (casos válidos) foi maior para o índice $a_{M F E L}\left(72 \%\right.$ dos casos) em relação ao índice $a_{f}(56 \%$ dos casos), e o parâmetro $F F L$ apresentou a maior aplicabilidade (89\% dos casos). A variabilidade do índice $a_{M F E L}$ foi menor que $10 \%$ na maior parte dos casos. 0 número de casos em que foi possível a obtenção de todos os parâmetros foi maior para os ensaios realizados a $25^{\circ} \mathrm{C}$. Além disso, ensaios realizados com materiais dentro da faixa de módulo de cisalhamento complexo de $12 \mathrm{MPa}$ a $60 \mathrm{MPa}$ apresentaram maior aplicabilidade dos índices $a_{M F E L}, a_{f}$ e $F F L$ do que ensaios realizados com materiais fora dessa faixa, como indica a Figura 9. A menor aplicabilidade dos índices para ensaios realizados nas temperaturas de $15{ }^{\circ} \mathrm{C}$ e $35^{\circ} \mathrm{C}$, bem como em materiais fora da faixa de $12 \mathrm{MPa}$ a 60 $\mathrm{MPa}$, pode ser explicada por problemas de falha de adesão entre a amostra e a placa, possível de acontecer em casos de rigidez elevada, ou fluxo de borda, que pode ocorrer em casos em que o material apresenta rigidez baixa, como indicado por Safaei e Castorena (2016). Pode-se suspeitar ainda da inadequação da geometria de $8 \mathrm{~mm}$ de diâmetro para realizar essas medidas em temperaturas mais baixas, tendo em vista que geometrias de menor diâmetro são comumente empregadas em ensaios com materiais de maior rigidez (seja por efeito térmico ou de 
envelhecimento) para contornar limitações relativas à capacidade de torque dos aparelhos. Esta última hipótese demanda estudos adicionais, por exemplo, empregando a geometria de $4 \mathrm{~mm}$. Medidas reológicas obtidas em materiais muito rígidos, nos quais a rigidez se aproxima da rigidez do próprio sistema de aplicação de carga, demandam a adequada correção da compliância do reômetro. Esta hipótese também demanda verificações adicionais, de modo a garantir que as medidas sejam precisas, isto é, que elas não estejam contaminadas pela compliância do aparelho.



(a)

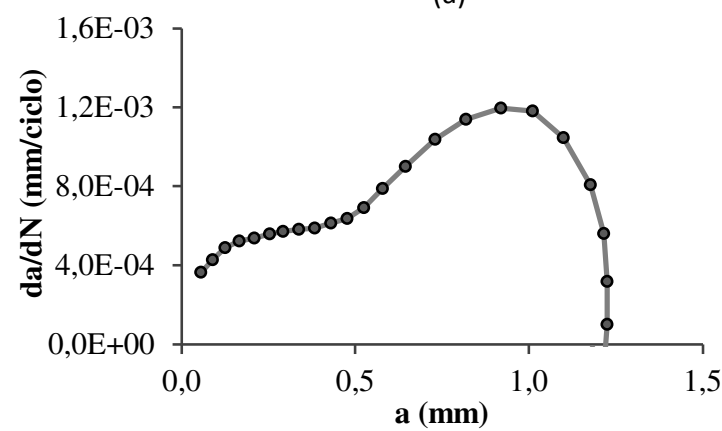

(c)

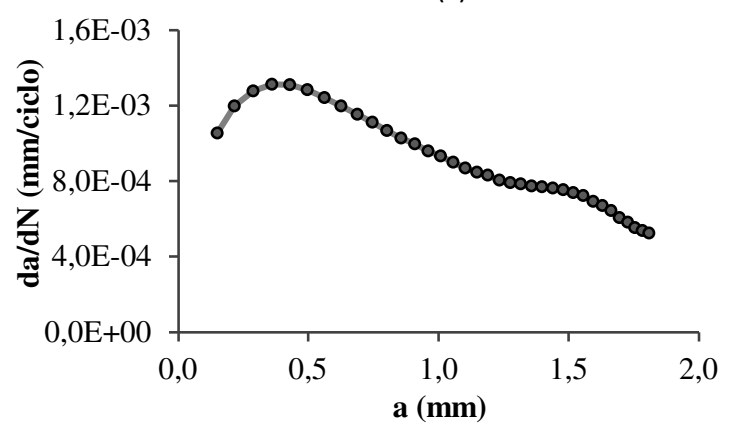

(e)

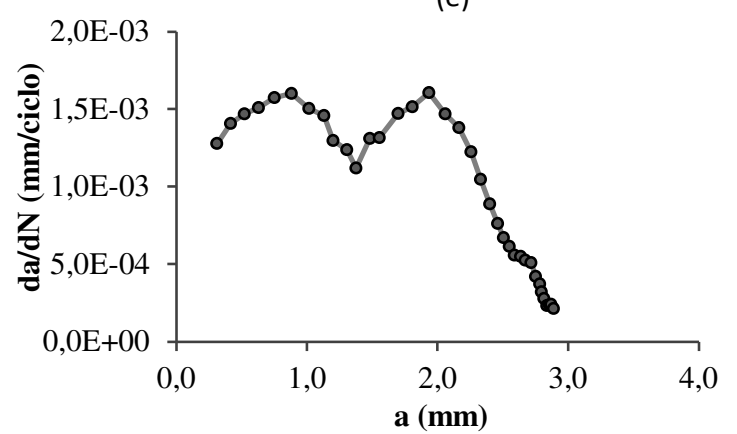

(g)

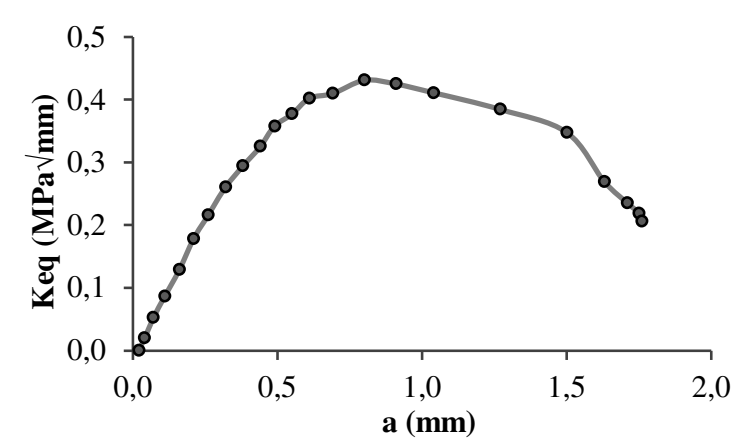

(b)

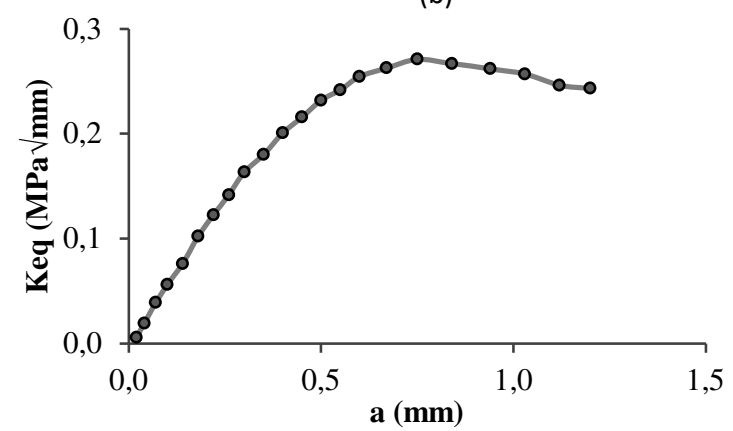

(d)

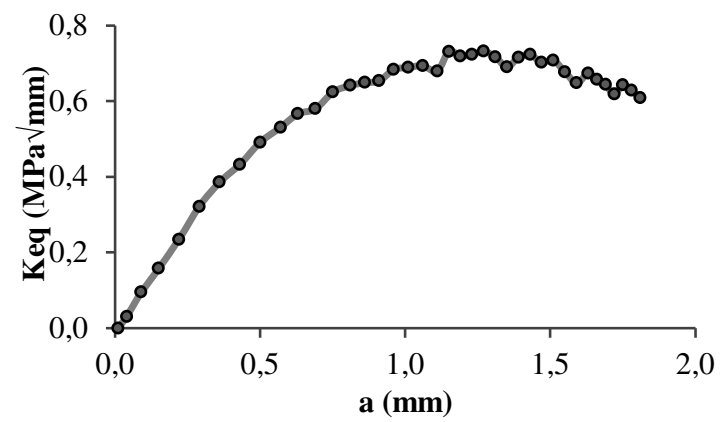

(f)

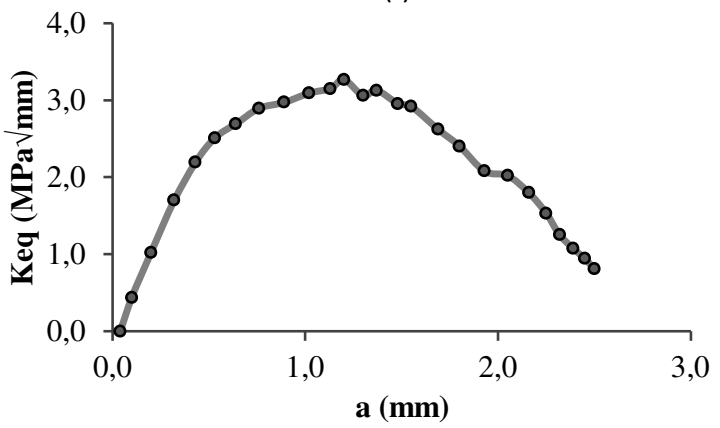

(h)

Figura 8. (a) Curva $d a / d N$ vs. $a$ e (b) curva $K_{e q}$ vs. $a$ para o material A a $35^{\circ} \mathrm{C}$, (c) curva $d a / d N$ vs. $a$ e (d) curva $K_{e q}$ vs. $a$ para o material B a $35^{\circ} \mathrm{C}$, (e) curva $d a / d N$ vs. $a$ e (f) curva $K_{\text {eq }}$ vs. $a$ para o material $\mathrm{C}$ a $25^{\circ} \mathrm{C}$, (g) curva $d a / d N$ vs. $a$ e (h) curva $K_{e q}$ vs. $a$ para o material E a $15^{\circ} \mathrm{C}$ 


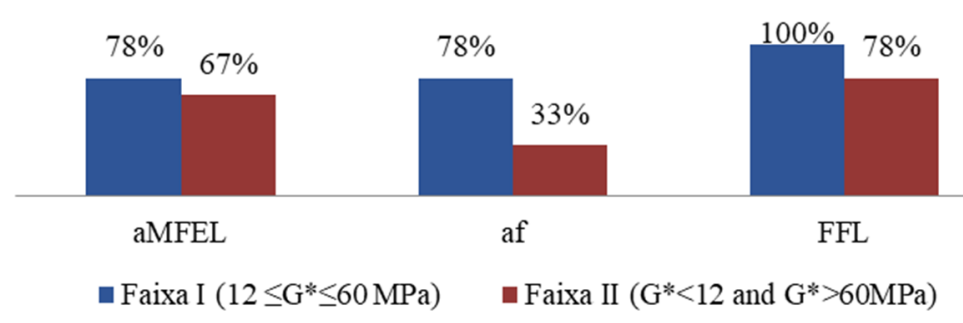

Figura 9. Aplicabilidade dos índices separada por faixa de valores de $G^{*}$

As Figuras 10 e 11 apresentam resultados dos ensaios $L A S$ realizados a $25^{\circ} \mathrm{C}$, na forma das curvas empregadas no cálculo dos índices $a_{f}$ e $a_{M F E L}$, respectivamente. Não foi possível determinar o índice $a_{f}$ do material $\mathrm{C}$, em virtude das peculiaridades da curva $d a / d N$ versus $a$ deste material, porém foi possível determinar com precisão o parâmetro $a_{M F E L}$. A Figura 12 apresenta os resultados do ensaio de varredura de tempo a $25{ }^{\circ} \mathrm{C}$ na forma das curvas de taxa de energia dissipada. A Figura 13 mostra as curvas de fadiga obtidas por meio da análise baseada na teoria VECD. A Figura 14 apresenta as correlações entre os parâmetros de fadiga analisados, com base nos ajustes lineares e seus respectivos coeficientes de determinação. 0 índice $a_{M F E L}$ apresentou correlação alta com o índice $a_{f}\left(\mathrm{R}^{2}=0,92\right)$ e correlação boa com o índice $F F L\left(\mathrm{R}^{2}=0,63\right)$, mas não apresentou correlação significativa com o parâmetro $N_{p 20}\left(\mathrm{R}^{2}=0,48\right)$. Da mesma forma, os índices $a_{f}$ e FFL não apresentaram correlação significativa com o parâmetro $N_{p 20}$. A Figura 15 apresenta um comparativo entre os parâmetros derivados do ensaio LAS e o parâmetro $N_{p 20}$ derivado do ensaio de varredura de tempo.

Embora não seja o objetivo primordial deste artigo comparar os materiais, os dados permitem inferir que o material D e o material C (ambos asfaltos modificados com polímero elastomérico) são mais tolerantes à fadiga, à luz do parâmetro a $M F E L$, e os materiais D e F (ambos asfaltos modificados com polímero elastomérico) possuem maior tolerância à fadiga segundo o parâmetro $a_{f}$. Com relação ao parâmetro $F F L$, o material E (asfalto de alto módulo) possui melhor resistência à fadiga, seguido pelo material D. Segundo o parâmetro $N_{p 20}$, o material C é o melhor em termos de resistência à fadiga, seguido pelo material E. Além disso, os materiais A e $\mathrm{B}$ (ambos asfaltos puros) se mostraram os piores em termos de tolerância à fadiga e de resistência à fadiga, o que foi indicado por todos os parâmetros analisados.

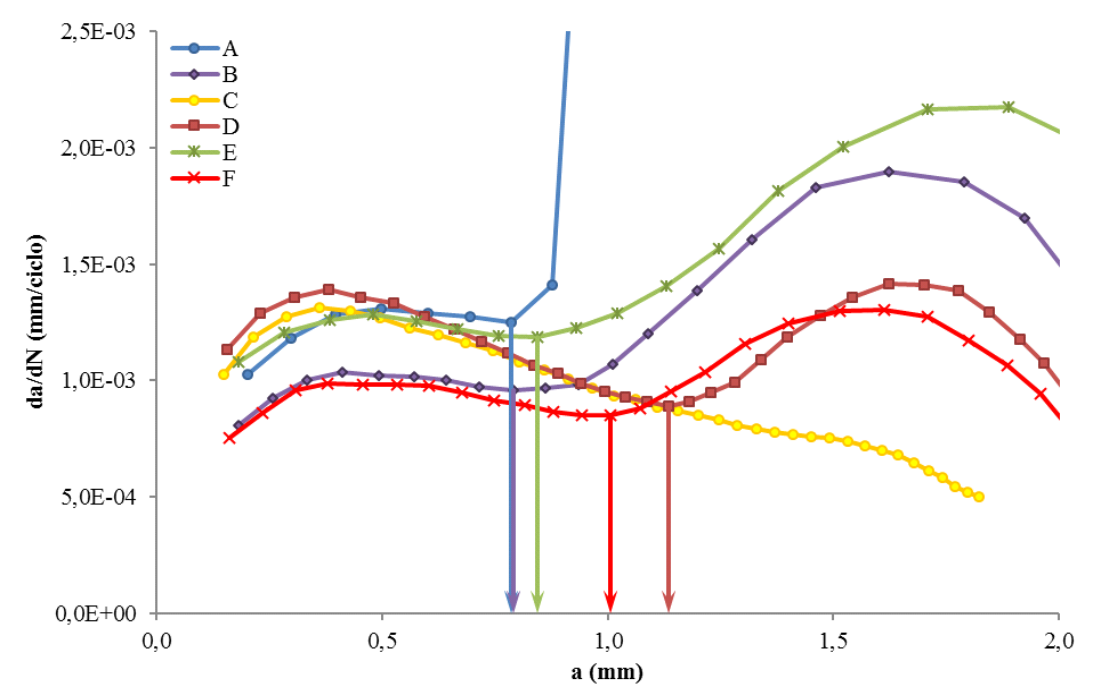

Figura 10. Curvas $d a / d N$ versus $a$ dos ensaios $L A S$ a $25^{\circ} \mathrm{C}$ de ligantes envelhecidos a longo prazo 


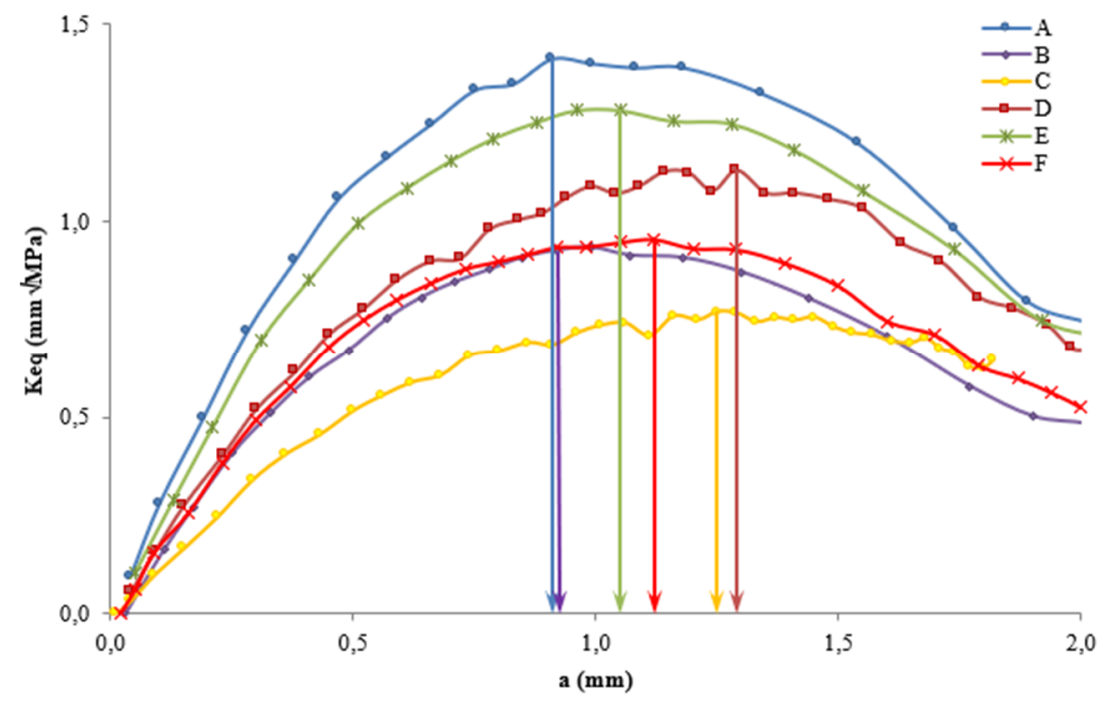

Figura 11: Curvas Keq versus $a$ dos ensaios $L A S$ a $25^{\circ} \mathrm{C}$ de ligantes envelhecidos a longo prazo

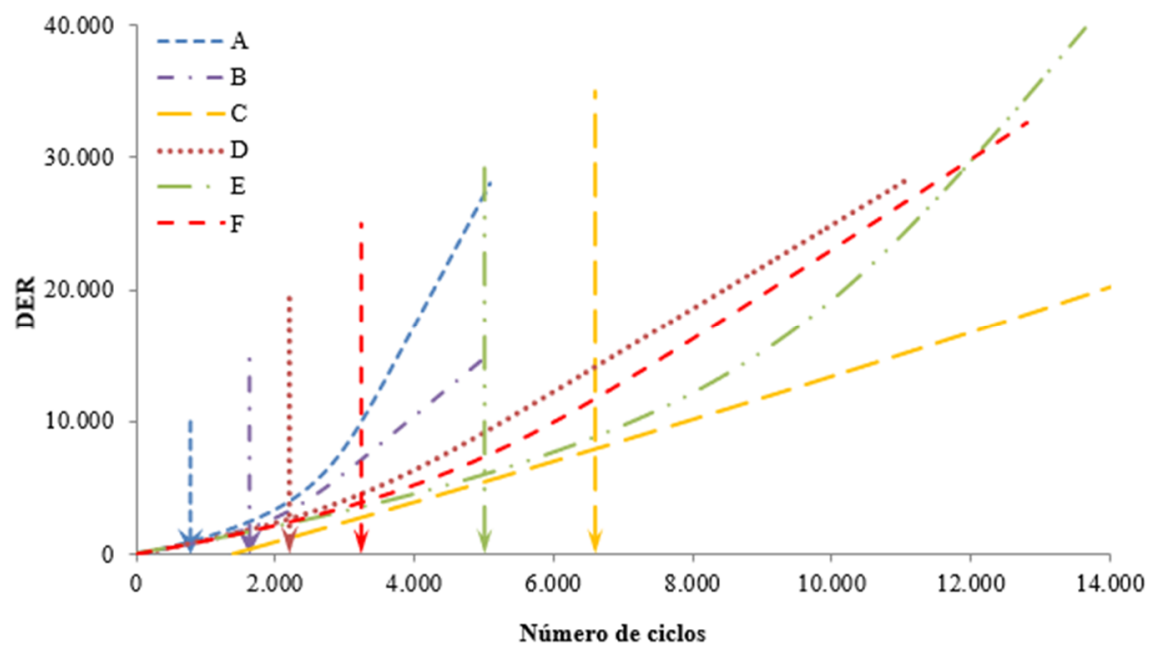

Figura 12: Variação da taxa de energia dissipada em função do número de ciclos para ensaios $L A S$ a $25{ }^{\circ} \mathrm{C}$ de ligantes envelhecidos a longo prazo

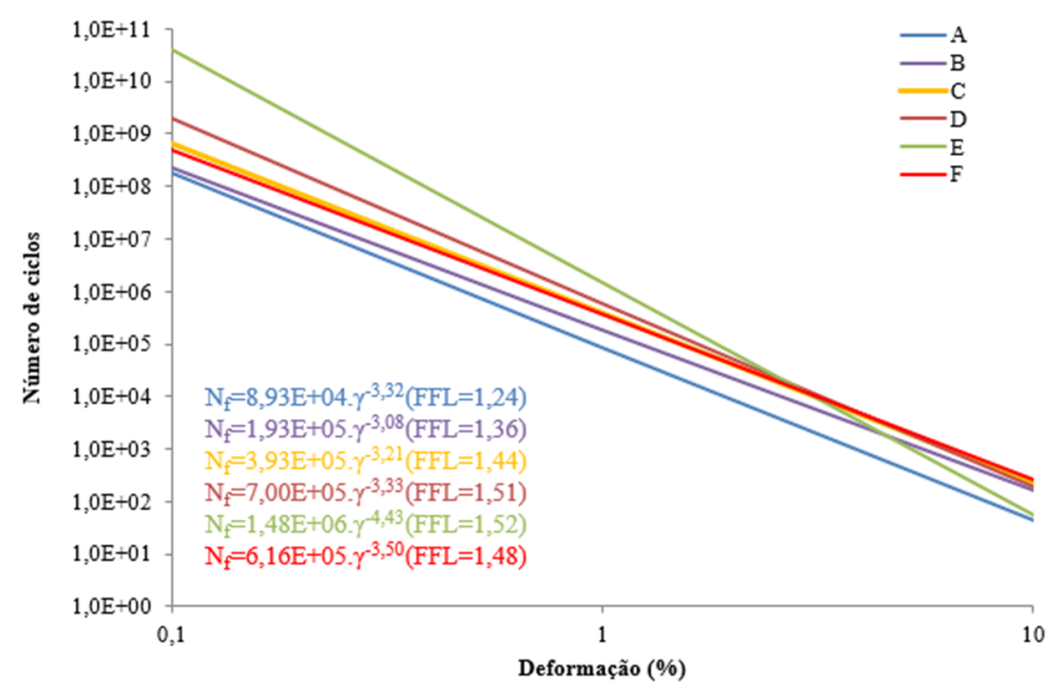

Figura 13: Curvas de fadiga dos ensaios $L A S$ a $25^{\circ} \mathrm{C}$ obtidas por meio da abordagem baseada na teoria VECD 


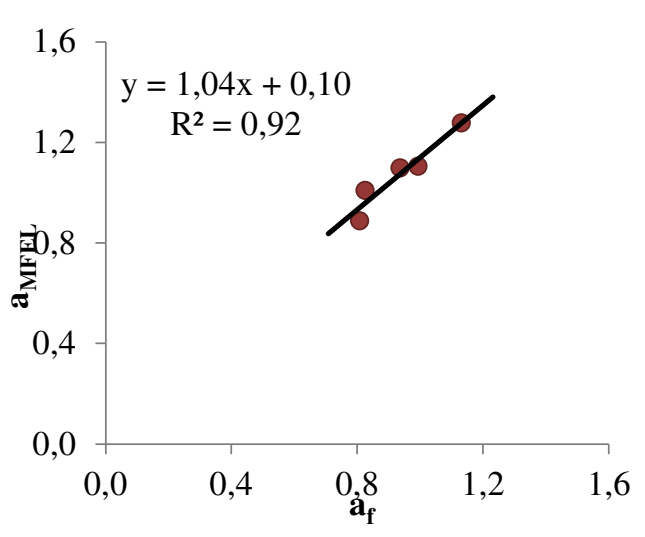

(a)



(c)

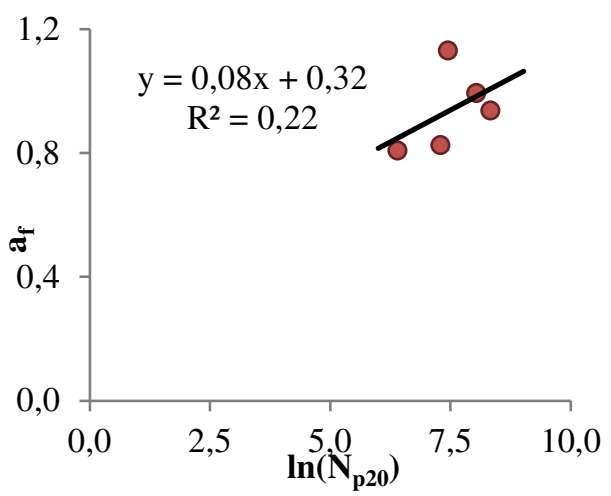

(e)

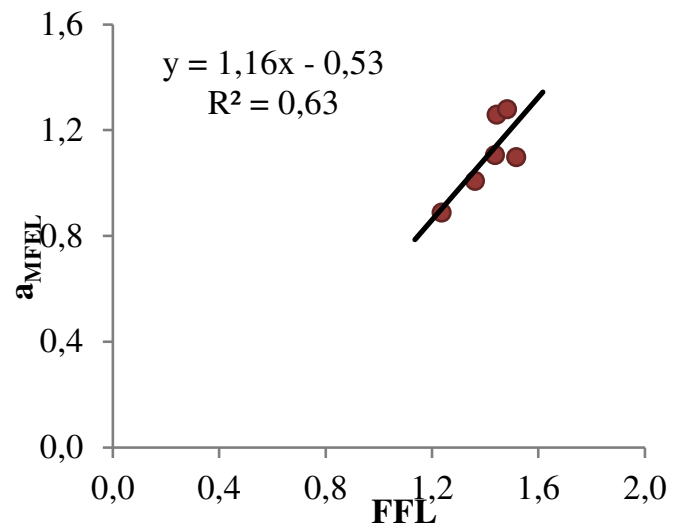

(b)

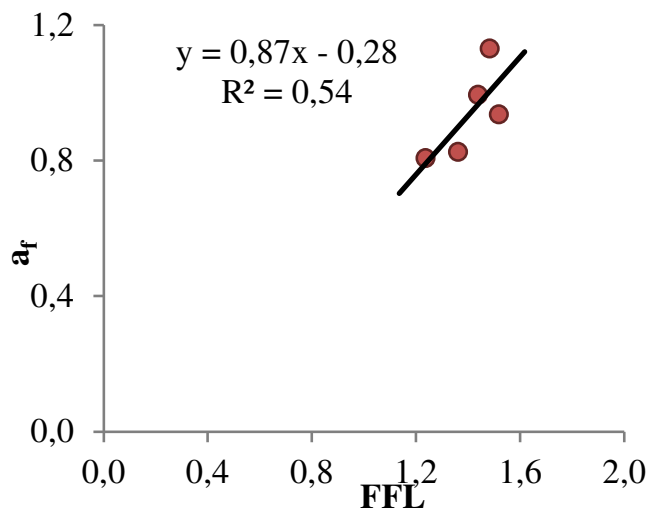

(d)

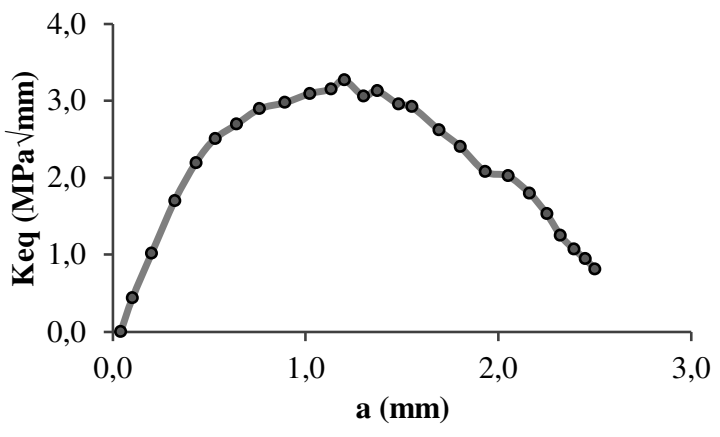

(f)

Figura 14: Correlações entre (a) $a_{M F E L}$ e $a_{f}$, (b) $a_{M F E L}$ e $F F L$, (c) $a_{M F E L}$ e $\ln \left(N_{p 20}\right)$, (d) $a_{f}$ e $F F L$, (e) $a_{f}$ e $\ln \left(N_{p 20}\right)$ e (f) $F F L$ e $\ln \left(N_{p 20}\right)$

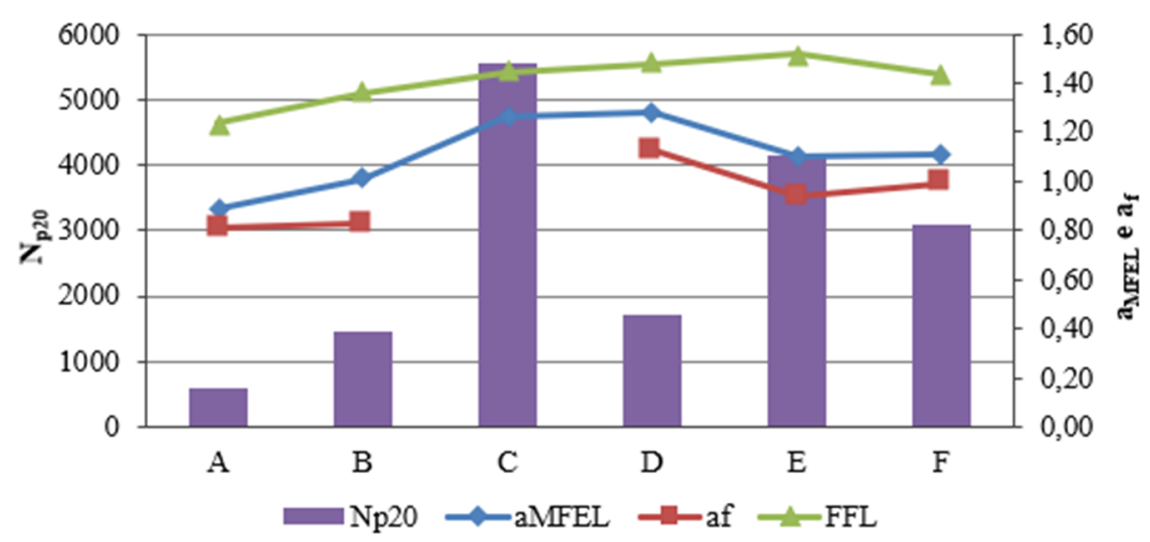

Figura 15: Comparativo entre os parâmetros $a_{M F E L}, a_{f}$ e $F F L$ e o parâmetro $N_{p 20}$ para ensaios a $25^{\circ} \mathrm{C}$ 
A Figura 16 apresenta a distribuição de tensões principais de tração em um exemplo de seção fissurada após a simulação no programa FRANC2D/L. A descontinuidade no material introduzida pela fissura gera uma concentração de tensão e deformação na ponta da fissura, que pode ser visualizada na aproximação da Figura 16. Dessa forma, a degradação mecânica do material ocorrerá nesta região, como representado pelo modelo numérico.

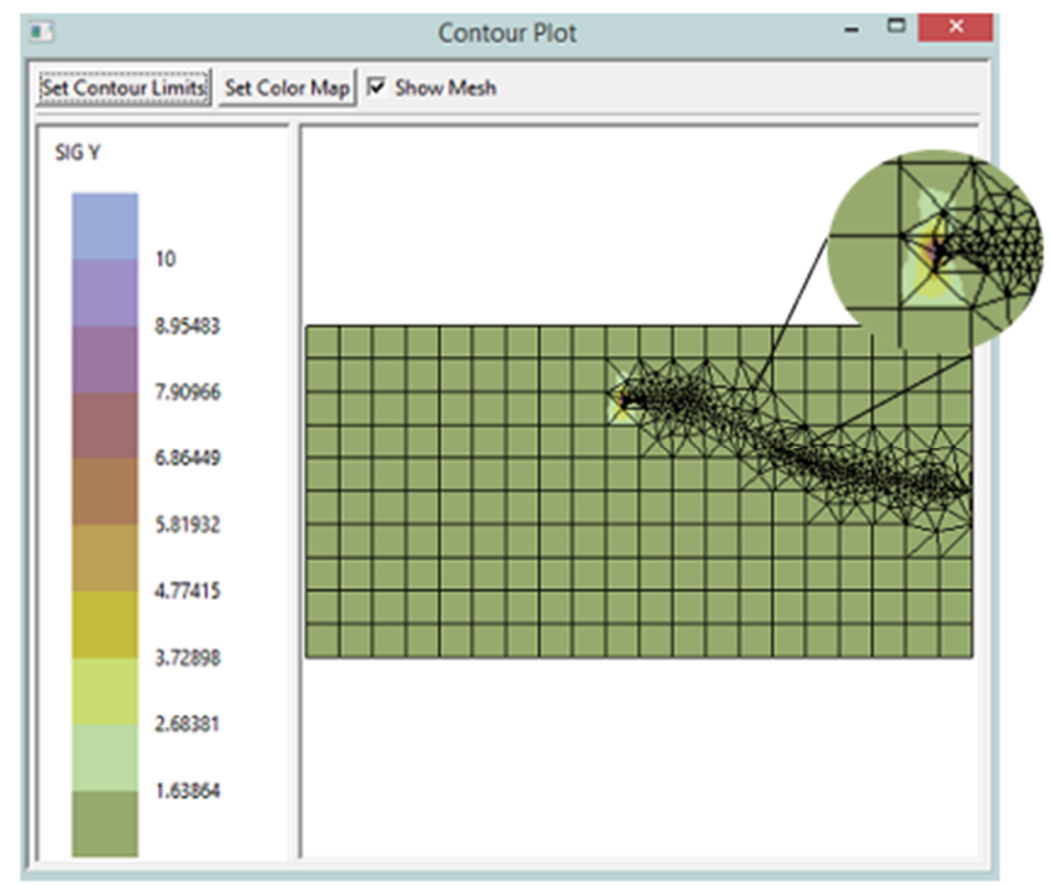

Figura 16. Distribuição de tensão principal de tração em seção após a simulação no programa FRANC2D/L

\section{CONCLUSÕES}

A análise dos resultados de ensaio $L A S$ por meio da abordagem baseada na mecânica da fratura elástico-linear apresentou resultados promissores. Os resultados obtidos neste estudo permitem formular as seguintes conclusões:

i. o software FRANC2D/L se apresenta como uma ferramenta útil para simular computacionalmente o ensaio $L A S$, no entanto a simulação feita com os valores de incremento de fissura calculados com base nos resultados de ensaio $L A S$ demanda bastante tempo;

ii. o índice $a_{M F E L}$ pôde ser obtido em um maior número de casos que o índice $a_{f}$, o que indica maior aplicabilidade do índice baseado na MFEL em relação à abordagem baseada na fratura; dentre os três índices analisados neste estudo, o índice FFL apresentou a maior aplicabilidade;

iii. o índice $a_{M F E L}$ apresentou boa correlação com o índice $a_{f}\left(\mathrm{R}^{2}=0,92\right)$ e com o índice $F F L$ $\left(\mathrm{R}^{2}=0,63\right)$, porém os índices $a_{M F E L}$ e $a_{f}$ não apresentaram correlação significativa com o parâmetro $N_{p 20}\left(\mathrm{R}^{2}=0,48\right.$ e $\mathrm{R}^{2}=0,22$, respectivamente); dentre os índices $a_{M F E L}, a_{f}$ e $F F L$, o índice $F F L$ foi o que apresentou maior correlação com o parâmetro $N_{p 20}\left(\mathrm{R}^{2}=0,67\right)$;

iv. os ensaios realizados com materiais sob condições tais que a rigidez esteja contida na faixa de $G^{*}$ de $12 \mathrm{MPa}$ a $60 \mathrm{MPa}$ apresentaram maior número de casos válidos para os três índices, o que reforça a indicação de que ensaios realizados com materiais cuja 
rigidez esteja fora dessa faixa estão sujeitos a problemas de instabilidade da amostra e perda de aderência entre o material e o aparelho;

v. o ordenamento dos materiais, tomando por base os resultados dos ensaios realizados a $25{ }^{\circ} \mathrm{C}$, indicou que os asfaltos $\mathrm{C}$ e $\mathrm{D}$ (ambos asfaltos modificados com polímero elastomérico) se mostraram os mais tolerantes à fadiga, à luz dos índices $a_{f}$ e $a_{M F E L}$, e também os mais resistentes à fadiga, à luz dos índices $F F L$ e $N_{p 20}$; os asfaltos puros (materiais $\mathrm{A} \mathrm{e}$ B) resultaram os menos tolerantes à fadiga e os menos resistentes à fadiga, à luz dos quatro parâmetros analisados neste estudo.

Apesar da correlação fraca entre os parâmetros $a_{M F E L}$ (derivado da teoria da mecânica da fratura elástico-linear) e $N_{p 20}$ (derivado do conceito de energia dissipada), o parâmetro $a_{M F E L}$ se apresenta como uma alternativa ao índice $a_{f}$, uma vez que também considera a macrofissuração que ocorre durante o ensaio $L A S$, além de ter apresentado maior aplicabilidade e maior correlação com o parâmetro $N_{p 20}$ que o índice $a_{f}$ (derivado da abordagem baseada na fratura). Os resultados deste estudo mostraram que a abordagem baseada na teoria do dano contínuo em meio viscoelástico (VECD) é a melhor alternativa para a caracterização dos materiais, em virtude da sua maior aplicabilidade e da maior correlação com o parâmetro $N_{p 20}$. Mesmo assim, pode-se afirmar que a aplicação da mecânica da fratura elástico-linear gerou resultados promissores, mesmo sem se fazer uma consideração precisa das propriedades viscoelásticas dos materiais. Os resultados aqui apresentados refletem a simplicidade e as limitações da teoria empregada, porém abrem caminhos para estudos que apliquem teorias mais apropriadas a materiais viscoelásticos, como a mecânica da fratura não-linear.

\section{AGRADECIMENTOS}

Os autores agradecem a Coordenação de Aperfeiçoamento de Pessoal de Nível Superior (CAPES) pela concessão de bolsa de estudos ao primeiro autor (processo n. 1680401).

\section{REFERÊNCIAS}

AASHTO (2014) Standard method of test for estimating damage tolerance of asphalt binders using the Linear Amplitude Sweep, AASHTO TP 101-14, Washington, DC.

Anderson, D. A. e T. W. Kennedy (1993) Development of SHRP binder specification (with discussion). Association of Asphalt Paving Technologists, v. 62, p. 481-507.

Anderson, D. A.; D. W. Christensen; H. U. Bahia; R. Dongre; M. G. Sharma; C. E. Antle e J. Button (1994) Binder Characterization and Evaluation Volume 3 : Physical Characterization, Report SHRP-A-369, Washington.

Anderson, D.; Y. Hir; M. Marasteanu; J. P. Planche; D. Martin e G. Gauthier (2001) Evaluation of fatigue criteria for asphalt binders. Transportation Research Record, v. 1766, p. 48-56. DOI:10.3141/1766-07

Bahia, H. U. e D. A. Anderson (1995) Strategic Highway Research Program binder rheological parameters: background and comparison with conventional properties. Transportation Research Record, v. 1488, p. 32-39.

Bahia, H. U.; D. I. Hanson; M. Zeng; H. Zhai; M. A. Kharti e R. M. Anderson (2001a) Characterization of modified asphalt binders in Superpave mix design. NCHRP report 459, National Academy Press, Washington, DC.

Bahia, H. U.; H. Zhai; M. Zeng; Y. Hu e P. Turner (2001b) Development of binder specification parameters based on characterization of damage behavior. Association of Asphalt Paving Technologists, v. 70, p. 442-470.

Bahia, H. U.; H. Wen e C. M. Johnson (2010) Developments in intermediate temperature binder fatigue specifications. Transportation Research Circular E-C147, p. 25-33. D0I:10.17226/22903

Bonnetti, K.; K. Nam e H. U. Bahia (2002) Measuring and defining fatigue behavior of asphalt binders. Transportation Research Record, v. 1810, p. 33-43. DOI:10.3141/1810-05

Christensen, D. W. e D. A. Anderson (1992) Interpretation of dynamic test data for paving grade asphalt cements. Association of Asphalt Paving Technologists, v. 61, p. 67-116.

FRANC2D/L. (2016) Cornell Fracture Group, Cornell University. Disponível em: <http://cfg.cornell.edu/software/>. (Acesso em 10/11/2019).

Griffith, A. A. (1921) The phenomena of rupture and flow in solids. Philosophical Transactions of The Royal Society of London, v. 221, p.163-198.

Hintz, C.; R. Velasquez; C. Johnson e H. U. Bahia (2011) Modification and validation of linear amplitude sweep test for binder fatigue specification. Transportation Research Record, v. 2207, p. 99-106. D0I:10.3141/2207-13 
Hintz, C. (2012) Understanding mechanisms leading to asphalt binder fatigue. Tese (doutorado). The University of Wisconsin, Madison.

Irwin, G. R. (1957) Analysis of stresses and strains near the end of a crack traversing a plate. Journal of Applied Mechanics, v. 24, n. 3, p. 361-364.

Johnson, C. M. (2010) Estimating asphalt binder fatigue resistance using an accelerated test method. Tese (doutorado). University of Wisconsin, Madison.

Kim, Y.; H. J. Lee; D. N. Little e Y. R. Kim (2006) A simple testing method to evaluate fatigue fracture and damage performance of asphalt mixtures. Association of Asphalt Paving Technologists, v. 75, p. 755-788.

Martins, A. T. (2014) Contribuição para a validação do ensaio de resistência ao dano por fadiga para ligantes asfálticos. Dissertação (mestrado). Universidade Federal do Rio de Janeiro, Rio de Janeiro, RJ.

Nascimento, L. A. H.; S. M. N. Rocha; E. H. Carlos; Y. R. Kim; M. Chacur e A. T. Martins (2014) Uso da mecânica do dano contínuo na caracterização de misturas asfálticas brasileiras. 21 Encontro de Asfalto, p. 1-14.

Nuñez, J. Y. M. (2013) Caracterização à fadiga de ligantes asfálticos modificados envelhecidos a curto e longo prazo. Dissertação (mestrado). Universidade de São Paulo, São Carlos.

Nuñez, J. Y. M.; E. D. Leonel e A. L. Faxina (2016) Fatigue characteristics of modified asphalt binders using fracture mechanics. Engineering Fracture Mechanics, v. 154, p. 1-11. D0I:10.1016/j.engfracmech.2016.01.001

Pamplona, T. F.; J. Y. M. Nuñez e A. L. Faxina (2014) Desenvolvimentos recentes em ensaios de fadiga em ligantes asfálticos. Transportes, v. 22, n. 3, p. 12-25. DOI:10.14295/transportes.v22i3.682

Pronk, A. C. e P. C. Hopman (1991) Energy dissipation: the leading factor of fatigue. Highway Research: Sharing the Benefits. The United States Strategic Highway Research Program, p. 255-267. London, England.

Sabouri, M.; D. Mirzaeian e A. Moniri (2018) Effectiveness of Linear Amplitude Sweep (LAS) asphalt binder test in predicting asphalt mixtures fatigue performance. Construction and Building Materials, v. 171, p. 281-290. DOI:10.1016/j.conbuildmat.2018.03.146

Safaei, F. e C. H. Castorena (2016) Temperature effects of Linear Amplitude Sweep testing and analysis. Transportation Research Record, v. 2574, p. 92-100. DOI:10.3141/2574-10

Shenoy, A. (2002) Fatigue testing and evaluation of asphalt binders using the dynamic shear rheometer. Journal of Testing and Evaluation, v. 30, n. 4, p. 303-312. DOI:10.1520/JTE12320J

Soenen, H.; C. de La Roche e P. Redelius (2003) Fatigue behaviour of bituminous materials: from binders to mixes. Road Materials and Pavement Design, v. 4, n. 1, p. 7-27. DOI:10.1080/14680629.2003.9689938

Timoshenko, S. P. e J. Gere (1983) Mecânica dos Sólidos. LTC Ed., v. 1, Rio de Janeiro.

Underwood, B. S. (2011) Multiscale constitutive modeling of asphalt concrete. Tese (doutorado). North Carolina State University, Raleigh, North Carolina. 